\title{
Research of the Effect of Services on the Aggregate Bandwidth of a Cell Radio Access Network UTRAN of UMTS Standard
}

\author{
Voropayeva Viktoriya* \\ Department of Automatic and Telecommunications, Donetsk National Technical University, Donetsk, Ukraine \\ *Corresponding author: Voropayeva@donntu.edu.ua
}

Received December 30, 2012; Revised January 19, 2013; Accepted February 26, 2013

\begin{abstract}
UMTS Terrestrial Radio Access Network (UTRAN) dimensioning has been of great interest among network researchers and planners due to the limit of cell bandwidth. This paper investigates the impact of service changes on the nature of radio interface UMTS bandwidth. Finally, OPNET simulation models are developed. The main purpose of modeling was to obtain the dependency of common capacities of the base station for the upward and downward directions on time. Different types of customers were used as the criterion for comparison the values obtained.
\end{abstract}

Keywords: bandwidth, radio access network, services, UMTS standard, download trend, upload trend

\section{Introduction}

Nowadays, telecommunication systems and networks of third generation $(3 \mathrm{G})$, which operate on the basis of wideband code division multiple access, are becoming more and more popular in the world. Their relative effectiveness in relation to the existing second generation systems (GSM, DAMPS) causes prospectivity of their current usage and in the near future. The fact that most of mobile services market continues to rely on traditional GSM-technology is caused by rather political and economic reasons, when network operators are not willing to part with well-proven technologies to provide custom services.

However, over the past 3-5 years there have been significant qualitative changes in the policies carried out by network operators, as it became clear that even advanced GSM-network (the introduction of technologies GPRS, EDGE, AMR) [1,2] cannot compete with the more advanced technology $3 \mathrm{G}$. In this case, the main problem was the possibility of co-operation of various systems under a single telecommunications system of the third generation, positioned as a global telecommunications system (UMTS-Universal Mobile Telecommunications System) and to develop common standards on its basis $[3,4]$.

At the beginning of its development, UMTS system borrowed many elements and functional principles of GSM system, and new and the most important solutions have been proposed in the field of radio access. The concept of UMTS provides an enhanced access technology (the broadband radio access). Wideband code division multiple access (WCDMA) is used for the implementation of the technology. WCDMA technology is based on the code separation between users while using the same frequency all over the radio network. Hence, the WCDMA based system is interference limited, and arises new planning aspects compared to more traditional FDMA or TDMA access technologies [5].

Each service makes new demands for the transmission path, carrying the information of a specific service. Some services are more demanding than others. Usually in the same network there are a lot of services and service requests from many users. Each service has its own requirements. Since network resources are limited, the task is to allocate enough resources for each request, no more and no less [6].

The main problem to be solved, which needs constant attention, is the total capacity of the cell with your service. To achieve a high level of functioning of quality multimedia network it is necessary to carry out careful network planning and to optimize the main parameters of the new conditions that arise during the operation.

To provide high quality services, mobile network operators continuously monitor the parameters of the real system, use methods of mathematical modeling and simulation.

The purpose of this paper is to study the effect of different types of services on the total capacity of the cellbased network on the basis of the universal telecommunication system UMTS.

\section{Architecture of the UMTS Network}

A new radio access network UTRAN was introduced for UMTS R99. UTRAN network technology is based on WCDMA, introduced in order to achieve a more efficient use of bandwidth in comparison with the methods used in 
GSM / GPRS (Figure 1). The network architecture consists of two main areas:

- Radio network subsystem (RNS);

- Basic network - packet switching domain (PS).

UTRAN network includes one or more of the RNS. Each RNS comprises a radio network controller (RNC) and one or more Node B. Nodes B are connected to the RNC through the interface Iub. Nodes B provide radio access to the network. The RNC each RNS can interact through the interface Iur.

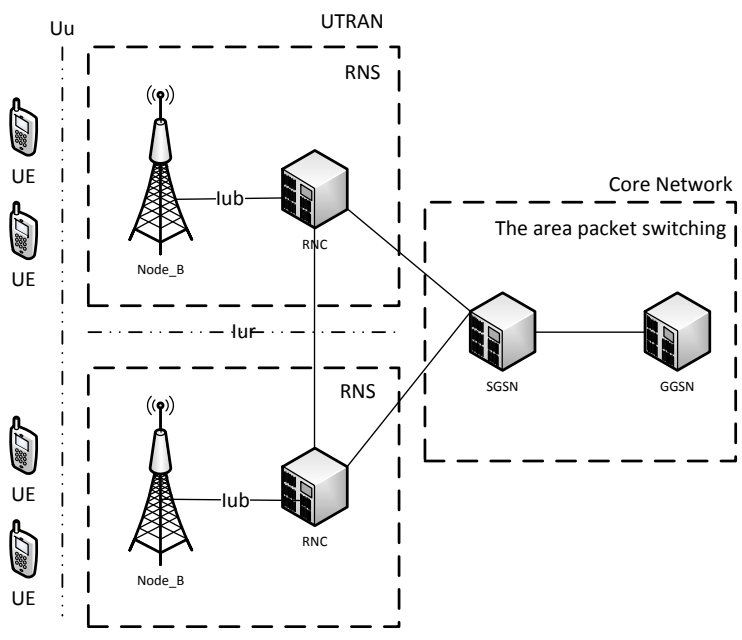

Figure 1. Architecture of the UMTS network

Radio network controller (RNC). Each RNC is responsible for managing radio resources of a set of cells. RNC is the equivalent BSC networks GSM / GPRS, but more self-governing. The roles of RNC in the UTRAN network may vary:

- Managing RNC. Each RNC is responsible for the resources of its set of cells and nodes B in the RNS. In this role, $\mathrm{RNC}$ is called managing RNC (CRNC).

- Serving RNC. For each connected UE RNC controllers may play an additional role: serving $\mathrm{RNC}$ (SRNC) which provides the connected UE radio resources.

- Drifting RNC. To minimize the impact of the handover, the RNC could play the third role: Drifting RNC (DRNC). DRNC provides ("gives for temporary use") resources to SRNC controller for a specific UE. Usually DRNC also act as SRNC (or DRNC) for other UE.

Node B. Provides transmission and reception of signals in one or more cells in the network like BTS in GSM network. Node B is also responsible for monitoring the power level of the inner loop.

User equipment (UE) is the equivalent of a mobile station (MS) in GSM, that is terminal, with the help of which a user can access the network. UE comprises mobile equipment (terminal) and universal service identification module (USIM). Mobile equipment is uniquely identified with the help of IMEI. To allow more upgrades, terminal equipment must have an application programming interface (API). USIM provides personal mobility, providing the user with access to services for which the subscriber is signed. In contrast to the SIM-card in a GSM USIM card can support a set of profiles. Each profile will have a specific purpose. It can be used to regulate the services available, depending on the capabilities of the terminal, in which the USIM card is installed. Both the user and the network can adjust the profiles.

The domain packet consists of the following elements:

1. Serving support node GPRS (SGSN). SGSN acts as a packet switch and router in the domain of PS core network. SGSN controls access to a mobile station to the network and routes packets to the correct BSC / RNC. It serves as the mobility management (MM), as does the MSC in the CS core network domain, such as registering the location of the adjustment zone routing (RAU) and paging. SGSN also handles the functions of privacy, such as authentication and encryption (between the MS / UE and SGSN).

2. Gateway Support Node GPRS (GGSN). GGSN acts as a packet router in the domain PS of the core network and is the gateway between routing IP packets to the mobile network UMTS and IP packet routing in fixed networks of the Internet. It carries out the packets transfer between networks, multimedia and IP-relevant SGSN, which currently serves the MS / UE. If MS changes SGSN during the standby mode, GGSN is used to buffer data packets. GGSN stores subscriber data for active MS / UE and performs the functions of privacy, such as firewalls and filtering $[3,4]$.

\section{Mathematical Model Studies}

The concept of load factor network UMTS have been taken as the basis of theoretical considerations. This factor allows quantifying the level of intra-system interference in UMTS channels and, ultimately, setting the specific conditions of permissible load on the network [5].

$\mathrm{S} / \mathrm{N}$ ratio for each user:

$$
\left(E_{b} / N_{0}\right)_{j}=\frac{W}{k_{j} R_{j}} \cdot \frac{P_{j}}{I_{\text {total }}-P_{j}}
$$

where $\mathrm{W}$ is the chip rate, $P_{j}$ is the received signal power from the user $\mathrm{j}, k_{j}$ is the activity factor of the user $\mathrm{j}$ ( $k=0.67$ - for a speech given $50 \%$ activity, and additional costs for intermittent mode of speech DTX, $k=1,0$ - for data), $R_{j}$ is the bit rate of the user $\mathrm{j}$, and $I_{\text {total }}$ is the total received wideband power including thermal noise power in the base station.

Solving for $P_{j}$ gives:

$$
P_{j}=\frac{1}{1+\frac{W}{\left(E_{b} / N_{0}\right)_{j} \cdot R_{j} \cdot k_{j}}} I_{\text {total }}
$$

We define $P_{j}=L_{j} \cdot I_{\text {total }}$ and obtain the load factor $L_{j}$ of one connection

$$
L_{j}=\frac{1}{1+\frac{W}{\left(E_{b} / N_{0}\right)_{j} \cdot R_{j} \cdot k_{j}}}
$$


The total received interference, excluding the thermal noise $P_{N}$, can be written as the sum of the received powers from all $\mathrm{N}$ users in the same cell:

$$
I_{\text {total }}-P_{N}=\sum_{j=1}^{N} P_{j}=\sum_{j=1}^{N} L_{j} \cdot I_{\text {total }}
$$

The noise rise is defined as the ratio of the total received wideband power to the noise power:

$$
\text { Noise_rise }=\frac{I_{\text {total }}}{P_{N}}
$$

and using Equation (4) we can obtain:

$$
\text { Noise_rise }=\frac{I_{\text {total }}}{P_{N}}=\frac{1}{1-\sum_{j=1}^{N} L_{j}}=\frac{1}{1-\eta_{U L}}
$$

where we have defined the load factor $\eta_{U L}=\sum_{j=1}^{N} L_{j}$

When $\eta_{U L}$ becomes close to 1 , the corresponding noise rise approaches infinity and the system has reached its pole capacity. Additionally, in the load factor the interference from the other cells must be taken into account by the ratio of other cell to own cell interference, $i$ :

$$
i=\frac{\text { other_cell_interference }}{\text { own_cell_interference }}
$$

The uplink load factor can then be written as:

$$
\eta_{U L}=(1+i) \sum_{j=1}^{N} L_{j}=(1+i) \sum_{j=1}^{N} \frac{1}{1+\frac{W}{\left(E_{b} / N_{0}\right)_{j} \cdot R_{j} \cdot v_{j}}}
$$

The load factor in the downward direction is defined similarly as in the previous case, although the parameters are somewhat different:

$$
\eta_{D L}=\sum_{j=1}^{N} v_{j} \cdot \frac{\left(E_{b} / N_{0}\right) j}{W / R_{j}} \cdot\left[\left(1-\alpha_{j}\right)+i_{j}\right]
$$

where $\alpha_{j}$ - coefficient of orthogonality of the $\mathrm{j}$ code channel, depending on the multipath in the channel [6].

\section{The Simulation Results}

Mobile UMTS network was built in the OPNET Modeler (Optimized Network Engineering Tool), which provides a convenient and easy-to-use platform for simulating large-scale networks [8]. OPNET Modeler is very popular commercial network simulation and analytical tool, which is used for network design, modeling and simulation. This program provides an integrated environment for simulation of communication networks and distributed systems. OPNET modeler provides a graphical editor interface to build models for various network entities from physical layer modulator to application processes [8].

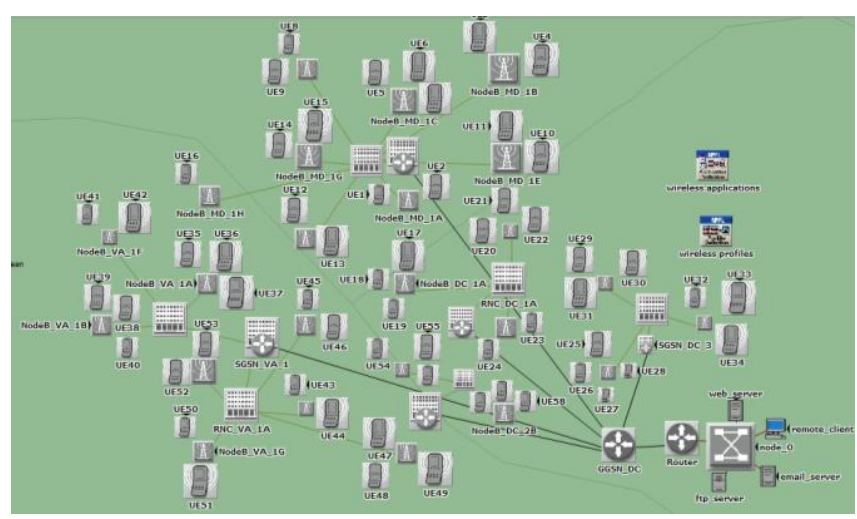

Figure 2. OPNET model of the UMTS network

The users have the possibilities to design and study many type of communication networks, devices, protocols and applications with flexibility and scalability. OPNET also provide very useful tools for simulations of the Radio Access Network UTRAN.

A comprehensive simulation model as shown in the Figure 2 has been developed by the author under the OPNET simulation environment. In this model three types of users are defined: ordinary mobile station (umts station), advanced workstations (umts wkstn) and servers (umts server). The mobile station includes the application layer, RLC / MAC layer, radio transmitter and receiver, one antenna and the protocols TCP (UDP) / IP.

4 types of users were selected for simulation:

- wireless_client that use e-mail(Light), Web Browsing (Light), File Transfer (Light), Voice over IP Call (PCM Quality);

- web that use Web Browsing (Light);

- e-mail that use e-mail(Light);

- ftp that use File Transfer (Light).

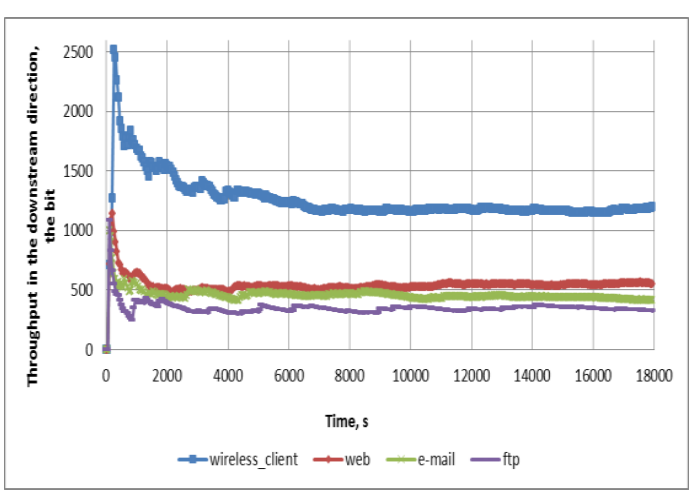

Figure 3. Download trend

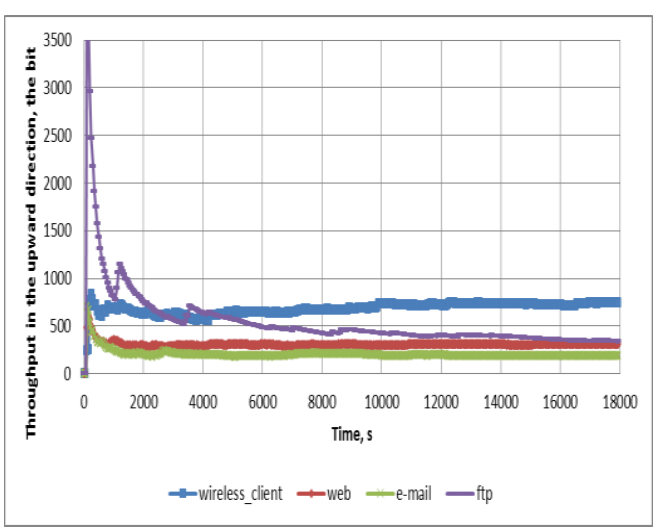

Figure 4. Upload trend 
In the modeling process types of subscribers will be changed to the following radio interface parameters:

- throughput in downlink and uplink directions to a BS;

- the total capacity in the downlink and uplink directions of the cell network.

While modeling, types of users in the network cell varied, the simulation was conducted for a limited period of time of 5 hours. Thus, we obtained the following relation (Figure 3 and Figure 4).

From these values it is clear that the maximum bandwidth allocated to the 'wireless_client' subscriber type. The reason for this is that these subscribers can simultaneously use several services: e-mail (Light), Web Browsing (Light), File Transfer (Light), Voice over IP Call (PCM Quality).

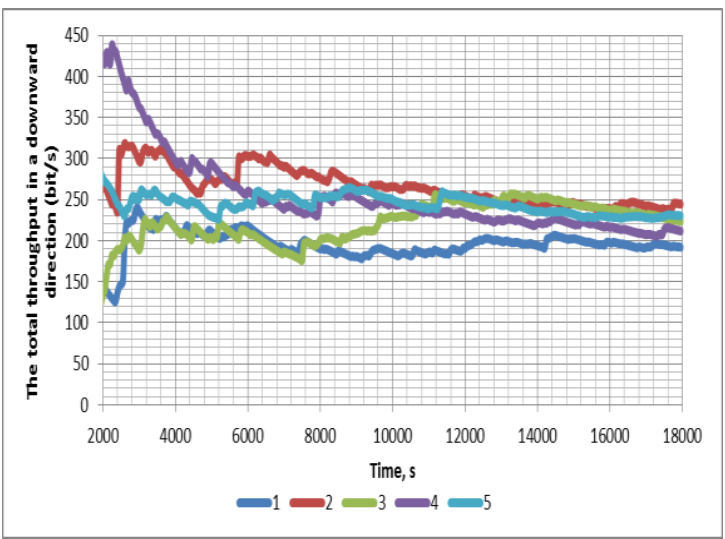

Figure 5. Dependence of the total capacity with different numbers of users

For customers who use only web bandwidth in a downward direction is approximately $600 \mathrm{bit} / \mathrm{s}$, while for 'wireless client' subscribers it is 1400 bits/s. The least demanding subscriber bandwidth users were ftp. For them bandwidth of 350 bits / s has been allocated. In total upstream bandwidth behaves somewhat differently. So email users were the least demanding of bandwidth 180 $\mathrm{bit} / \mathrm{s}$ in contrast to the downward direction.

Figure 5 shows the effect of the number of subscribers for a total average throughput of the cell.

To obtain a complete picture of the influence of services on the bandwidth we use the dependencies in Figure 6 and Figure 7, which reflect the impact of services on the bandwidth at the radio access network UTRAN.

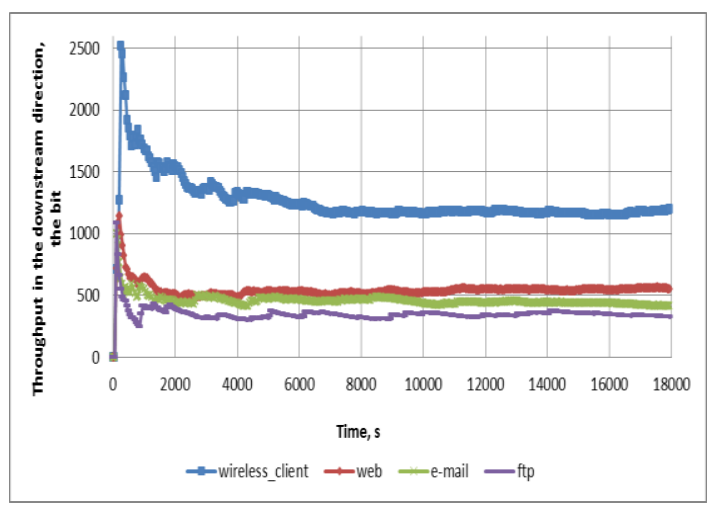

Figure 6. Download trend

Thus, these figures show that at the level of the radio access network UTRAN the trend of bandwidth values for different types of services remains unchanged. This is explained by the principles of building UMTS network, which are divided into two aspects: the physical realization of the individual network units and the formation of functional connections between them.

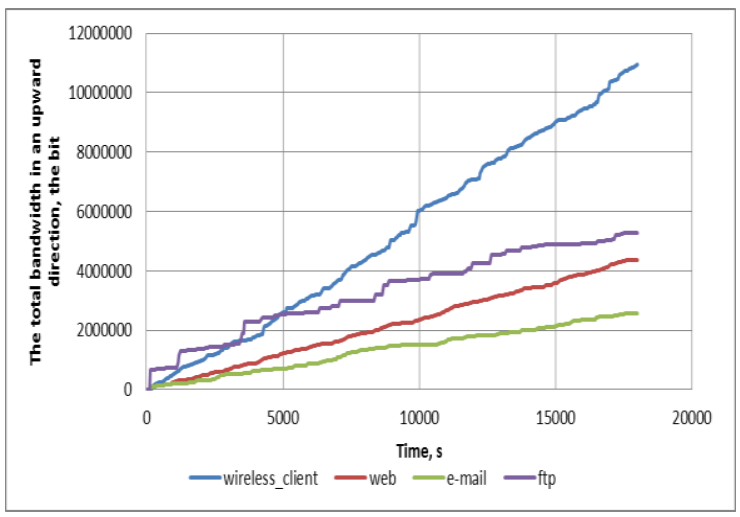

Figure 7. Upload trend

\section{Conclusion}

In this paper the impact of service changes on the nature of radio interface UMTS bandwidth has been researched. The main purpose of modeling was to obtain the dependency of common capacities of the base station for the upward and downward directions on time. Different types of customers were used as the criterion for comparison the values obtained. Types of customers meant different sets of services. During the simulation it turned out that the most demanding of bandwidth were subscribers who used multiple services simultaneously. Thus, the described features, obviously, need to be taken into account during the preliminary planning and construction of networks.

\section{References}

[1] Mishra, A. R.., Advanced Cellular Network Planning and Optimisation: 2G/2.5G/3G...Evolution to 4G, John Wiley \& Sons, Ltd, Chichester, UK, 2006.

[2] Neonakis Aggelou, G.; Tafazolli, R.; "On the relaying capability of next-generation GSM cellular networks," Personal Communications, IEEE, 8(1): 40-47, 2001.

[3] Timo Halonen, Javier Romero, Juan Melero, GSM, GPRS and EDGE Performance: Evolution Towards 3G/UMTS, 2nd ed, John Wiley \& Sons, Ltd, Chichester, UK, 2003.

[4] Holma H., al. WCDMA for UMTS: Radio Access for Third Generation Mobile Communications, 2nd ed., John Wiley and Sons Inc., West Sussex, England, 2002.

[5] Moulsley, T.J.; "Throughput of high speed downlink packet access for UMTS," 3G Mobile Communication Technologies, 2001. Second International Conference on (Conf. Publ. No. 477), 363367, 2001.

[6] Voropayeva, V.Y.; Krasikova, A.S. "Evaluating the influence of mobile user movement speed upon the throughput of a UMTS network cell," in Microwave and Telecommunication Technology (CriMiCo), 2012 22nd International Crimean Conference, 269$270,2012$.

[7] X.Li, W. Cheng, A.Timm-Giel, and C. Gorg, "Modeling IP-based UTRAN for UMTS in OPNET", distinguished paper award, in Proc. OPNETWORK 2007, September, 2007, Washington DC, USA, 2007.

[8] X. Li, S. Li, C. Gorg and A. Timm-Giel, "Traffic Modeling and Characterization for UTRAN," in Proc. $4^{\text {th }}$ International Conference on Wired/Wireless Internet Communications, Bern Switzerland, 2006. 